TBW negatively correlated with age $(\rho=-0.25, \mathrm{p}=0.02)$, disease duration $(\rho=-0.30$, $p=0.005)$, BMI $(\rho=-0.78, p=0.001)$ and the ocular component of the ESSPRI $(\rho=-0.28, p=0.01)$, but not with the NSWSF or the ESSPRI oral component. When we compared the patients in the $25 \%$ percentile (group with the lowest $\%$ of water)

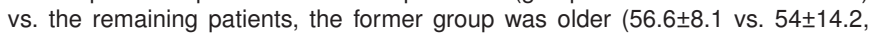
$p=0.02$ ), with longer disease duration $(12.4 \pm 5.9$ vs. $10.8 \pm 7.12, p=0.03)$, lower scores at the Schirmer test (1 (range $0-8)$ vs. 2 (range $0-9$ ), $p=0.01$ ), higher BMI $(31.1 \pm 5.1$ vs. $23.7 \pm 2.9, p=0.001)$ as well as with higher ESSPRI ocular domain scores $(8.3 \pm 1.4$ vs. $6.7 \pm 2.5, p=0.007)$. With the linear regression analysis, the variables that remained associated with the TBW were disease duration $(\beta-0.22$, $p=0.001)$, BMI $(\beta-0.76, p<0.001)$ and the ocular domain of the ESSPRI $(\beta-0.15$, $\mathrm{p}<0.001)$.

Conclusions: Patients with PSS had similar TBW percentage than controls. However among patients with PSS, the TBW had a negative correlation with the intensity of ocular symptoms independently of disease duration, age and BMI. References:

[1] Guyton, Arthur C. (2006). Textbook of Medical Physiology (11th ed.). Philadelphia: W.B. Elsevier Saunders. p. 295. ISBN 0-7216-0240-1.

Disclosure of Interest: None declared

DOI: 10.1136/annrheumdis-2017-eular.2388

\section{AB0500 TOLERABILITY, EFFICACY AND IMMUNOGENICITY OF 23-VALENT PNEUMOCOCCAL VACCINE IN SLE PATIENTS}

G.M. Tarasova, B.S. Belov, M.S. Sergeeva, S.K. Soloviev, E.A. Aseeva, N.G. Klukvina, T.V. Popkova, E.N. Alexandrova, A.A. Novikov, M.V. Cherkasova. V.A.Nasonova Research Institute of Rheumatology, Moscow, Russian Federation

Background: Concurrent infections turn out to be the second leading cause of death in systemic lupus erythematosus (SLE) pts after SLE per se. Immunization of SLE pts with pneumococcal vaccine is an important prophylactic approach to prevent severe lower respiratory tract (LRT) infections in SLE pts.

Objectives: To study the relevance of 23-valent pneumococcal vaccine for immunization of SLE pts.

Methods: The study included 30 SLE pts, 27 females, 3 males, aged $19-62$ y. Duration of follow up (FUP) was 12 months in 24 pts, and 7-10 months - in 6 pts. High disease activity at the time of immunization was documented in 1 patient, low activity - in 20 pts, moderate - in 4 pts, and remission - in 5. 29 pts were treated with glucocorticosteroids (GCs), 23 - with hydroxychloroquine, 14 pts - with cytostatic (CS) agents. Twelve pts were on biological diseasemodifying antirheumatic drugs (bDMARDs). One dose $(0,5 \mathrm{ml}$ ) of 23-valent polysaccharide pneumococcal vaccine was administered subcutaneously. The duration of FUP was 7-12 months. Control visits were scheduled as follows: at baseline (Visit 1), at 1st, 3rd, and 12th months (Visit 4) after immunization. Standard clinical examination and lab tests, including blood immunology, were performed at each visit. Vaccine immunogenicity was evaluated based on the level of serum antibodies (AT) to Streptococcus pneumoniae capsular polysaccharide (VaccZymeTM PCP Ig 2 panels (The Binding Site Ltd, Birmingham, UK)) - 4 times during 1 year.

Results: No post-immunization complications were seen in $11(36,7 \%)$ pts, local reactions of varying intensity lasting from 2 to 7 days were documented in $18(60 \%)$ pts. One patient $(3,3 \%)$ developed the local type III hypersensitivity reaction known as Arthus phenomenon. All symptoms subsided within 7 days after administration of antihistaminic agents and local GCs. Not a single vaccinationrelated SLE exacerbation episode was documented in 24 pts during the FUP. Significant ( $\geq 2$-fold vs baseline) increase of serum AT levels to $S$. pneumoniae polysaccharide was observed during the FUP (Table).

In $10(41,7 \%)$ out of 24 pts ("non-responders") more than 2-fold increase of antiS. pneumoniae ATs was not achieved by 12th month of FUP. Among them 7 $(70 \%)$ pts were receiving bDMARDs. $4(28,6 \%)$ out of 14 "responders " were also treated with bDMARDs.

Non-severe pneumonia was documented in 2 out of 24 pts within 1 year after vaccination; both cases successfully resolved after 7 - and 5-days of oral antibiotic treatment in an out-patient setting. Both pts had episodes of pneumonia in past medical history. One of them had SLE-induced interstitial lung disease. This patient was treated with GCs, mycophenolate mofetil, and rituximab, no post-vaccination response was documented. The second patient had two previous pneumonia episodes, she demonstrated 4-5-fold increase in anti- S. pneumoniae AT titre; her therapy included GCs $(10 \mathrm{mg} /$ day $)$ and hydroxychloroquine. There were no clinical or radiological symptoms of pneumonia in remaining 22 pts during the whole FUP.

\begin{tabular}{|c|c|c|c|c|}
\hline Visit & Visit 1 (baseline) & Visit 2 (1 mo $)$ & Visit $3(3 \mathrm{mo})$ & Visit 4 (12 mo) \\
\hline $\begin{array}{c}\text { AT } \\
\text { concentration } \\
\text { Mel }\end{array}$ & $\begin{array}{c}97,4^{*} \\
(68,0 ; 128,1)\end{array}$ & $\begin{array}{c}407,8^{b} \\
(231,2 ; 488,8)\end{array}$ & $\begin{array}{c}301,8 \\
(164,3 ; 424,6)\end{array}$ & $\begin{array}{c}265,5^{d} \\
(120,0 ; 438,7)\end{array}$ \\
\hline & & b-a, $p=0,00006$ & $c-2, p=0,00008$ & $\mathrm{~d}-\mathrm{a}, p=0,002$ \\
\hline
\end{tabular}

Conclusions: Obtained results are indicative of good tolerability, safety and immunogenicity of 23-valent pneumococcal vaccine in SLE pts. Further studies are necessary for more comprehensive evaluation of vaccine clinical efficacy.

Disclosure of Interest: None declared

DOI: 10.1136/annrheumdis-2017-eular.2347

\section{AB0501 THE ASSOCIATION BETWEEN GLUCOCORTICOIDS AND DAMAGE ACCRUAL IN PATIENTS WITH SLE USING GLUCOCORTICOID FOR LONG-TERM}

G. Wang, X. Li, Z. Dou, W. Wang, R. Liu. Rheumatology and Immunology, Anhui Provincial Hospital Affiliated to Anhui Medical University, Hefei, China

Background: Systemic lupus erythematosus (SLE) is a chronic systemic autoimmune disease characterized by a relapsing-remitting course. Long-term prognosis of SLE patients remains poor [1]. Due to the effect of potent anti-inflammatory and immunosuppressive, glucocorticoids (GCs) remain the cornerstone of treatment in SLE. However, GCs produce several adverse reactions, most are time and dose dependent, limiting their clinical usefulness. Increased longevity with prolonged exposure to GCs and inflammatory insults might contribute to organ damage accrual, which retards further improvement of survival in these patients [2]. Assessment the extent of organ damage caused by SLE has been considered an important part of the assessment of prognosis. The Systemic Lupus International Collaborating Clinics/American College of Rheumatology (SLICC/ACR) Damage Index (SDI) is a validated instrument designed to measure irreversible damage resulting from SLE disease activity and its treatment. The study of damage accrual in patients with SLE caused by the long-term treatment of GCs is still not clear.In a large SLE cohort, followed prospectively, we determined to investigate the association between damage accrual with GCs, both cumulative prednisone dose and high-dose prednisone. The results of our study could shed more light on the risk/benefit ratio of GCs in long-term maintenance treatment with SLE patients.

Objectives: To evaluate the association between long-term glucocorticoids use and damage accrual in patients with systemic lupus erythematosus.

Methods: Medical records of 535 SLE patients from Department of Rheumatology and immunology of Anhui Provincial Hospital were reviewed. 512 patients were femal. The cohort's mean age was $38.27 \pm 12.84$ years with mean disease duration of $7.33 \pm 5.75$ years. Their Systemic Lupus International Collaborating Clinics/American College of Rheumatology Damage Index (SDI) scores were noted. (1) Univariate analysis and multivariable regression analysis were performed to determine factors associated with SDI. (2) Analysis were also performed to determine damage associated with cumulative prednisone dose and high-dose of prednisone (exposure to prednisone at a dosage of $60 \mathrm{mg} /$ day for $1 \mathrm{month}$ ). Results: (1) Among 535 patients in our cohort only 5 paitents $(0.9 \%)$ had never been treated with glucocorticoids. A total of 192 patients $(35.9 \%)$ had been treated with high dose of prednisone. In addition, $86.9 \%$ of patients had been treated with hydroxychloroquine. (2) The highest organ damage was musculoskeletal $(n=79,14.8 \%)$, followed by skin damage $(n=35,6.5 \%)$ and renal $(n=28,5.2 \%)$. Ninety patients were diagnosed with hypertension. (3) SDI scores were associated with age of onset, exposure to high-dose prednisone, hypertension.(4) Cumulative prednisone dose was associated with osteoporosis, osteonecrosis and hypertension; exposure to high-dose prednisone was associated with osteonecrosis, lupus nephritis and hypertension.

Conclusions: Long-term taking prednisone predicted damage accrual. The most common damage was osteoporosis, osteonecrosis and hypertension.

References:

[1] Lopez R, Davidson JE, Beeby MD, et al. Lupus disease activity and the risk of subsequent organ damage and mortality in a large lupus cohort[J]. Rheumatology. 2012,51(3):491-8.

[2] Mak A, Isenberg DA, Lau CS. Global trends, potential mechanisms and early detection of organ damage in SLE[J]. Nature reviews Rheumatology. 2013,9(5):301-10.

Disclosure of Interest: None declared

DOI: 10.1136/annrheumdis-2017-eular.3931

\section{AB0502 STRONG REDUCTION OF ANTI-MÜLLERIAN HORMONE IN SYSTEMIC LUPUS ERYTHEMATOSUS WOMAN OF REPRODUCTIVE AGE}

C. Li, J. Xie, X. Wang, X. Liu, G. Chen. Division of Rheumatology and Clinical Immunology, the First Affiliated Hospital of Guangzhou University of Chinese Medicine, Guangzhou, China

Background: Systemic lupus erythematosus (SLE) is a clinically autoimmune disease characterized by production of autoantibodies and immune complex deposition. That induces multiple organ damages such as nephritis, pneumonitis and central nervous system (CNS) lupus et al. Moreover, SLE which mainly occurs in reproductive woman could threaten ovarian function. In recent years, ovarian reserve dysfunction in SLE are attracting increasing attentions. Especially cyclophosphamide (CYC) therapy was already well known as a higher risk to result in premature ovarian failure (POF) 1,2. However, few research has been performed in association between SLE itself and ovarian reserve. POF which is a critical cause of secondary amenorrhea and infertility still has not been treated effectively until now. Accordingly, an early precaution is important to make an informed decision about impaired ovarian reserve in SLE.

Objectives: To study the association between systemic lupus erythematosus (SLE) itself and strongly reduced Anti-Müllerian Hormone (AMH) values.

Methods: SLE women during reproductive ages 18-40 years were recruited compared with age-matched healthy controls (HC). AMH Levels and its relationship to clinical parameters and disease activity were investigated. 